\title{
Criado, Ignacio. 2016. Nuevas tendencias en la gestión pública. Innovación abierta, gobernanza inteligente y tecnologías sociales en unas administraciones públicas colaborativas. Madrid: Instituto Nacional de Administración Pública
}

Edgar Alejandro Ruvalcaba Gómez ${ }^{1}$

Fecha de recepción: 15 de agosto del 2017

Fecha de aceptación: 15 de agosto del 2017

\begin{abstract}
"Power does not reside in institutions, not even the state or large corporations.
It is located in the networks that structure society"
\end{abstract}

Manuel Castells.

uevas tendencias en la gestión pública es un libro editado por el Instituto Nacional de
Administración Pública (INAP) de España, que a lo largo de doce capítulos nos conec-
ta con casos de vanguardia, los cuales dan cuenta de administraciones públicas que emprenden su camino hacia la innovación y uso de las nuevas tecnologías. Los casos y estudios presentados, giran en torno a un nuevo paradigma de gestión pública conceptualizado como gobierno abierto.

Académicos y funcionarios públicos con experiencia en el tema y ocupados de hacer estudios son los autores que comparten sus trabajos en esta obra, la cual es resultado de dos mesas de trabajo en sendos Congresos sobre ciencia política y administración pública. Por un lado el grupo de trabajo Nuevos Enfoques en la Gestión Pública, reunido para el XII Congreso Español de Ciencia Política y de la Administración, celebrado en San Sebastián, 13 al 15 de julio de 2015; y por otro lado, el grupo de trabajo “¿Hacia unas administraciones públicas sociales?”, dentro del V Congreso Internacional en Gobierno, Administración y Políticas Públicas del GIGAPP, celebrado en Madrid, 29 de septiembre al 2 de octubre de 2015). 
Los capítulos presentados resaltan el panorama emergente de la administración pública desde una perspectiva de investigación y otra bastante operativa en administraciones innovadoras dentro del sector público, posicionando al texto como un referente dentro del campo de las transformaciones gubernamentales y el papel que asumen las nuevas tecnologías, principalmente en el ámbito hispano.

Un factor común encontrado en los capítulos son las dinámicas colaborativas aplicadas y orientadas a empoderar el rol del ciudadano y que aportan valor innovador a la gestión pública.

En el recorrer de los planteamientos emergen conceptos trascendentales: gobernanza inteligente, redes sociales, innovación pública, transparencia, colaboración, coproducción de servicios, gobierno electrónico, participación 2.0 y sobre todo gobierno abierto. Dichos conceptos son grandes temas en las agendas de los gobiernos que están emigrando a nuevos modelos de gestión, mismos que requieren ser estudiados y analizados profundamente. El texto ofrece una vinculación necesaria de la teoría con casos prácticos, ofreciendo un aporte en el camino a consolidar buenas prácticas y desarrollar teoría que expliquen los nuevos tiempos de la gestión pública.

El libro aborda cuatro grandes bloques: el primero caracterizado por un debate teórico, que reflexiona y dialoga la temática general de la innovación en el sector público, siendo éste bloque, elemento de orientación para entender la relevancia y pertinencia de libro en su conjunto. El segundo bloque está caracterizado por temáticas orientadas a sistema macros de gobernanza y enfoques de gestión vinculados al gobierno abierto, orientados a políticas de transparencia o seguridad inteligente. Un tercer bloque analiza las redes sociales y las grandes plataformas en el sector público, lo cual permite reflejar un estado del arte de estas herramientas en los gobiernos. Y por último un cuarto bloque de corte más institucionalista que se ocupa principalmente de las dinámicas de participación ciudadana y principalmente destacando el uso de las nuevas tecnologías como base instrumental para su consolidación.

El libro comienza con un breve resumen y presentación de los autores, seguido por un prólogo de Manuel Villoria, experto en temas de gestión pública e innovación administrativa, quien reflexiona en torno a la transformación de lo político y social, y la evolución de la gestión pública hasta conectar de forma aguda con el marco general de la temática del libro. Posteriormente Ignacio Criado, coordinador de la obra, hace una introducción en la que describe el sentido y relevancia del libro en la actualidad.

El primer capítulo corresponde a J. Ignacio Criado quien muestra un recorrido por la situación más actual de la gestión pública contemporánea, sus principales debates teóricos y conceptuales; el capítulo hace una conexión teórica con los grandes modelos organizacionales de gestión y administración pública (administración pública tradicional, nueva gestión pública o gobernanza pública), destacando la nueva era de la información y el potencial uso de las nuevas tecnologías que han penetrado la sociedad. Encontramos un fino análisis, no solo teórico sino también reflexivo en donde se plantean las ideas una gobernanza pública inteligente, dentro de nuevas dinámicas que están transformando la forma de hacer gobierno.

En el siguiente capítulo, Alberto Núñez expone un análisis global de las limitaciones y condicionantes sobre reforma en España, la tradición administrativa y su evolución transformadora; concluyendo que existe una falta de visión reformista del sistema administrativo; sin embargo hay grandes cambios latentes en la gestión pública.

Olga Ramírez y Miquel Salvador desarrollan una discusión en torno al proceso transformador que ha sufrido el gobierno digital hasta llegar al gobierno abierto. Desde un marco analítico organizacional se 
consideran dos experiencias de innovación en los que se observa cambio institucional, y los autores asocian tales cambios al crecimiento de políticas de transparencia y participación entre diferentes sectores.

Seguidamente Ignacio Criado y Julián Villorde se enfocan en la herramienta wiki dentro de un nuevo constructo de las administraciones públicas colaborativas. El análisis considera rasgos de la teoría de gestión del conocimiento e inteligencia colectiva para desarrollar el estudio de cuatro casos de éxito que implementan herramientas wikis. Ambos dan prueba de los aportes en la gestión del conocimiento e inteligencia colectiva.

En el quinto capítulo, Rafael Valenzuela hace un fino análisis para resaltar que el gobierno abierto genera condiciones para considerar la pertinencia de la implementación de políticas de seguridad inteligente. El capítulo destaca las características objetivas de factores evaluables, lo que da un paso hacia un proceso integral y transformador para poder adaptar un sistema complejo de seguridad al enfoque de gobierno abierto.

Tuve el honor de participar junto con Ignacio Criado en la elaboración de un capítulo que explora la temática de transparencia dentro la literatura internacional más relevante sobre gobierno abierto a modo de meta-análisis. Se presenta una estrategia analítica que permite aproximarse a la orientación de la transparencia gubernamental y mostrar sus atributos que le caracterizan dentro de una literatura que aborda el paradigma de gobierno abierto, en un capítulo que sienta bases para desarrollar estudios que den paso al análisis las políticas del tema.

Posteriormente se abordan las redes sociales digitales dentro de administraciones públicas, su función y retos. De este modo, Joan Balcells, Albert Padró-Solanet e Iván Serrano, nos ofrecen algunas dimensiones de las redes sociales y un análisis empírico de twitter en ayuntamientos catalanes, concluyendo que el éxito de estas redes no solo depende de los gestores, sino del apoyo estratégico de los dirigentes de primer nivel y la implicación de la organización gubernamental.

Abraham Lira desarrolla y analiza en el siguiente capítulo, una experiencia de la Agencia de Gestión Urbana de la Ciudad de México en Twitter donde muestra el potencial de la red social para aumentar la interacción y participación ciudadana. Un análisis referente en uso de twitter para los gobiernos que se están involucrando en el uso de redes.

En la misma línea Mercedes Díaz, proporciona datos empíricos resultado de un estudio en las universidades públicas españolas, donde se abordan cuestiones sobre la incorporación, la motivación y los factores del uso de las redes sociales de las universidades de España. Un capítulo que arroja evidencia que invita a la profundización del estudio, que da a conocer el impacto y uso de las redes sociales digitales.

En otro capítulo, Jesús Palomar nos presenta un análisis de empleados públicos dentro de las redes sociales y herramientas digitales, a partir de dos casos: la Escola d'Administració Pública de Catalunya y la Diputación de Barcelona. En ellos, considera el autor que la formación y capacitación de los empleados públicos son imprescindibles para evolucionar a nuevas formas de gobernanza como los planteados en el texto.

Los últimos dos capítulos del libro desarrollan casos de éxito de proyectos de participación ciudadana. Primero, se encuentra el texto de Koldobike Uriarte Ruiz de Eguino, Javier Bikandi Irazabal, Goizalde Atxutegi y Gotzon Bernaola Ariño, El Libro Blanco de Democracia y Participación Ciudadana para Euskadi documenta una práctica innovadora de participación, en la que desglosa la odisea democrática por construir un modelo de gobernanza, partiendo de una hoja en blanco y una apertura participativa. 
Y por último se presenta un estudio del Ayuntamiento de Alcobendas que gira entorno a las nuevas herramientas 2.0, para materializar la participación ciudadana. El caso presentado por Javier Arteaga e Ignacio Criado, revela datos que muestran los beneficios de estas herramientas a nivel local, lo que permite proyectar nuevas rutas transformadoras de la participación ciudadana.

A lo largo del libro se encuentra una idea transversal que Ignacio Criado deja ver en la introducción, y se refiere a la imposibilidad de entender la gestión pública de hoy en día como un espacio estático o fuera de la sociedad en red. Criado construye un argumento en el que refleja la intencionalidad de sepultar la vieja y tradicional gestión pública, una gestión que no aspira a la digitalización, participación y transparencia gubernamental como ejes rectores dentro del aparato administrativo.

Los capítulos que incluyen la obra van orientados a enaltecer las virtudes de un sistema público colaborativo que permita la co-creación de políticas públicas entre los diferentes sectores sociales, y la aspiración de lo que Criado menciona, como el anhelo de una gobernanza más inteligente por parte de las administraciones públicas. Este potente pensamiento lo vemos concentrado en la siguiente cita: “...ya no es que tengamos que plantearnos una gestión pública en la sociedad red, sino que ahora solo cabe hablar de una gestión pública en red(es), en otras palabras, una gobernanza inteligente”. 